\title{
How smart is peptide receptor radionuclide therapy of neuroendocrine tumors especially in the salvage setting? The clinician's perspective
}

\author{
Vikas Prasad • Winfried Brenner • Irvin M. Modlin
}

Published online: 6 November 2013

(C) Springer-Verlag Berlin Heidelberg 2013

In the recent past there has been a substantial growth in the understanding of cancer biology with a consequent opening of a Pandora's box of scientific knowledge and advance in the biological perception of the disease. Paradoxically, this indepth, sun-burst-like knowledge has fogged the several established management protocols rather than clearing the doubts. The 'knowledge' that the hitherto established and proven therapeutics do not only target cancer cells but also 'normal' cells has shifted the attention more onto toxicity.

To overcome the toxicity related to the 'generalized' treatment approach medical scientists across the world have gleefully (and possibly gullibly) accepted targeted therapy and the 'theranostic concept'. This evolved approach should eventually, supposedly, tackle the intelligent cancer cells which have successfully challenged 'Darwin's Theory of Evolution' by replacing the normal 'chosen' cells of the best creation and evolved creature of nature. Paradoxically survival of the fittest in this instance leads to extinction of the organism! This has generated a new intellectual theocracy whereby new knowledge has been embraced with almost religious zeal and rigorous assessment diminished in the face of fervor as we seek to reverse a biological Darwinian conundrum. As a consequence a note of caution needs to be placed here because the knowledge of the scientific medical community about neoplasia is juvenile, whereas the cancer cells, by the time they are diagnosed, are evolved and are up to 40 years old [1]. In effect they are the adults in the evolutionary cascade, and thereby not only challenging but

V. Prasad $(\bowtie) \cdot$ W. Brenner

Department of Nuclear Medicine, Campus Virchow-Klinikum,

Charité University Hospital, Berlin, Germany

e-mail: vikas.prasad@charite.de

I. M. Modlin

Department of Surgery, Yale University School of Medicine, New Haven, CT, USA also winning the natural selection process which decrees that "growth and spread is to the strongest". In consideration of such (very old) philosophy, direct implementation of the promising but not matured theranostic concept in a patient with aggressive cancer, with a relatively shorter life expectancy, might not have a significant adverse influence on an already poor quality of life. The problem however achieves a different degree of proportionality when the concept is translated to the treatment of an indolent tumor associated with a relatively good overall survival following the initial diagnosis. Neuroendocrine tumors (NETs) are a group of such tumors, whose incidence and prevalence appear to be increasing at an alarming rate, partially due to highly advanced diagnostic methods and raised awareness [2].

Nuclear medicine is the first exponent of the theranostic concept dating back to early 1950s. The classical example is the use of radioiodine for diagnosis and therapy of various benign and malignant thyroid diseases targeting the thyroid cell sodium iodine transporters. A similar analogy is the identification of the overexpression of somatostatin receptors on NETs. This has enabled nuclear medicine physicians to deal with the complex and heterogeneous tumor by addressing the neoplastic overexpression of particular subclasses of somatostatin receptor. Thus somatostatin receptor-based molecular imaging has become established as an essential tool in the management of NET. Alternatively however, peptide receptor radionuclide therapy (PRRT), using peptides such as DOTATATE or DOTATOC, despite the fact that numerous retrospective (often single-center) studies during the last 10 years have provided evidence of success, has led to controversial discussions amongst oncologists, surgeons and gastroenterologists. The critical points of discussion include toxicity (hematotoxicity and nephrotoxicity) rather than the efficacy of the therapy. Objective response rates range from $4 \%$ to $30 \%$ with significant increases in progression-free survival (PFS) compared to established approved therapy regimens [3]. 
In order to better understand this concern and overcome this push back from the nonnuclear medicine community one has to analyze the problem inside out or using Jabberwockian logic (a reasonable assessment of unreasonable information). Most patients with NET treated with PRRT, except in a few specialized centers, are followed up outside the domain of nuclear medicine. The duration of follow-up plays a significant role because (a) patients with metastasized NET generally have good to very good overall survival of more than 5-10 years from the time of first diagnosis, and (b) the major toxicities of PRRT are mostly chronic appearing several months after the first therapy. The other reason why the toxicity of PRRT is so critically discussed is the awareness that patients with a relatively low tumor burden, having somatostatin receptor positive lesion(s), if left untreated, or if only symptomatically treated, have a median time to progression (TTP) of 6-14.3 months [4]. The presence of the 'target', in this case somatostatin receptors on the tumor cells therefore may not justify treatment with targeted radionuclides. It is extremely important to define the timing of the PRRT in relation to other available therapeutic strategies such as surgical resection, somatostatin receptor analogs for symptom/antitumor therapy, locoregional therapy options, chemotherapy and 'wait and watch'. Indeed, most current experts (those who evolved in a therapeutic milieu in which PRRT was a distant planet) advocate the use of PRRT as a second- or third-line therapy option, i.e. treating only NET patients with progressive disease, thereby providing acceptable justification of the benefit to toxicity ratio [5].

These precepts are based upon information in the published literature that high-grade nephrotoxicity of PRRT ranges from $1 \%$ to $10 \%$ and can therefore dramatically reduce quality of life. Similarly severe hematotoxicity such as myelodysplastic syndrome (MDS) which has an occurrence ranging from $1 \%$ to $2 \%$ can be life-threatening [6-9]. Based upon dosimetry data and experience accumulated over the past 15 years, several patient-related coexisting risk factors compounding the toxicity of PRRT have been identified. These include age, diabetes mellitus, arterial hypertension and prior chemotherapy, and should be carefully evaluated when deciding on the administered activity for PRRT [10]. In addition a consideration of nonpatient-related factors such as the choice of radionuclide $\left({ }^{177} \mathrm{Lu}\right.$ vs. $\left.{ }^{90} \mathrm{Y}\right)$ and the interval between successive therapy cycles, route of administration and the utility of appropriate nephroprotection regimens renders the matter even more complex. Precautionary measures including adequate hydration before and after PRRT, stringent hygiene after PRRT (avoid urinary tract infections) and less stressful work during the 4-6 weeks after PRRT may help to overcome some of the acute side effects of PRRT. The incidence of chronic side effects can be optimally reduced (if clinically feasible) if a time interval of at least 36 months is maintained between PRRT and chemotherapy, especially 5-FU and platinum-based regimens. The experience of the responsible nuclear medicine physicians and the sequence of therapies also appear to play major roles.

In this issue of the EJNMMI, Sabet et al. report that salvage PRRT is safe and effective [11]. This is not only exciting and good news for individuals who progress after initial PRRT cycles but is also very important information for treating clinicians. The physician confronted with the question of what to do next in a patient with progressive disease without many safe, approved alternative therapy options requires guidance and reassurance that is provided by this analysis. The authors report that salvage PRRT with ${ }^{177} \mathrm{Lu}$-DOTATATE led to a complete response in 1 patient $(3.0 \%)$, a partial response in $6(18.2 \%)$, a minor response in 1 (3\%), stable disease in 14 $(42.4 \%)$ and progressive disease in $11(33.3 \%)$. Median PFS from the commencement of salvage therapy was 13 months, and patients with a history of durable PFS after initial PRRT tended to have long-lasting PFS after salvage treatment. None of the patients developed high-grade nephrotoxicity or MDS during follow-up. Relevant albeit reversible hematotoxicity (grade $3 / 4$ ) occurred in 7 patients $(21.2 \%)$. The cumulative administered activity was not associated with an increased incidence of hematotoxicity.

The salvage therapy experience (in 33 patients) reported by van Essen et al. similarly confirms that salvage therapy is effective. Their data analysis demonstrated a median TTP after regular therapy of 27 months [12]. In four patients, the intended cumulative dose of $14.8 \mathrm{GBq}$ in two cycles was not achieved (two had progressive disease, two had long-lasting thrombocytopenia). Hematological toxicity grade 3 was observed in four patients, and grade 4 in one patient. The median follow-up time was 16 months (range 1-40 months). No kidney failure or MDS was observed. Renewed tumor regression was observed in eight patients (two with partial remission, six with a minor response), and eight patients exhibited stable disease. The median TTP was 17 months. Treatment outcome was less favorable in patients with a short TTP after regular cycles.

Treatment effects in pancreatic NETs are similar to those in other gastroenteropancreatic NETs. Most interestingly the group from Rotterdam found that global health status/quality of life (GHS/QOL), Karnofsky performance status, and symptoms all improved significantly after ${ }^{177}$ Lu-octreotate therapy. Furthermore, there was no significant decrease in QOL in those who were asymptomatic prior to therapy. Of particular note is the observation that individuals with suboptimal GHS/QOL scores or symptoms before therapy experienced a clinically significant improvement. These results show that ${ }^{177} \mathrm{Lu}$-octreotate therapy is not only effective in reducing tumor size but also prolongs overall survival and improves patients' self-assessed QOL [13].

Although MDS was not observed either by the Rotterdam group or by Sabet et al., a total of 11 patients reported in the 
PubMed-listed literature out of more than 3,000 patients treated with PRRT developed MDS. Compared to chemotherapy regimens for other tumor entities, this is a very low number; however, one cannot ignore this serious side effect in the context of NET, especially if it occurs in the salvage setting.

The responsibility to allay the fears related to PRRT lies collectively in the hands of nuclear medicine physicians across the world. As a group it is necessary not only to objectively develop the required information but also to disseminate this to a diverse group of oncology-related physicians to both educate and reassure them. In this respect guidance would be obtained from the way oncologists plan their studies. Thus it is abundantly evident that monocenter retrospective analyses are no longer adequate and only prospective multicenter studies such as NETTER-1 can help answer critical questions with a sufficiently high level of evidence. Realistically, however, the prospective identification of molecular markers of cell (renal or myeloid) toxicity and the identification of genomic indicators of susceptibility would be critical steps forward. This 21 st century molecular approach rather than seeking event associations based simply upon incidence and prevalence alone would substantially advance the field and prospectively define risk or lead to early identification rather than await organ failure.

Similarly, this novel and important concept of salvage therapy of NET needs to be tested in a prospective manner in order to reproduce the results at an evidence-based level. The cost and time associated with such prospective studies remain important issues, but there is no other 'shorter and quicker path' to reimbursement of treatment costs by health insurance providers. Clearly the time and energy invested in undertaking retrospective analyses has failed to meet the criteria of any 'significance' in the rigorous eyes of the FDA and EMA. Appropriate and open communication with surgeons, oncologists and gastroenterologists is the call of the hour ("ask not for whom the bell tolls") to interfere in a successful and timely manner with communication networks between cancer cells and normal cells before they evolve further [14] and become unsalvageable and resistant to internal targeted irradiation. If PRRT is to enter the management mainstream and become a true "ready for prime-time therapy", then significant reconsideration of the value of our product and an objective scientific basis for pitching it has to be promulgated both by ourselves and our parent organizations. It should be remembered that once Pandora's box was emptied all that was left inside was hope!

\section{References}

1. O’Shaughnessy JA, Kelloff GJ, Gordon GB, Dannenberg AJ, Hong WK, Fabian CJ, et al. Treatment and prevention of intraepithelial neoplasia: an important target for accelerated new agent development. Clin Cancer Res. 2002;8:314-46.

2. Modlin IM, Lye KD, Kidd M. A 5-decade analysis of 13,715 carcinoid tumors. Cancer. 2003;97:934-59. doi:10.1002/cncr.11105.

3. Zaknun JJ, Bodei L, Mueller-Brand J, Pavel ME, Baum RP, Horsch $\mathrm{D}$, et al. The joint IAEA, EANM, and SNMMI practical guidance on peptide receptor radionuclide therapy (PRRNT) in neuroendocrine tumours. Eur J Nucl Med Mol Imaging. 2013;40:800-16. doi:10. 1007/s00259-012-2330-6.

4. Rinke A, Muller HH, Schade-Brittinger C, Klose KJ, Barth P, Wied $\mathrm{M}$, et al. Placebo-controlled, double-blind, prospective, randomized study on the effect of octreotide LAR in the control of tumor growth in patients with metastatic neuroendocrine midgut tumors: a report from the PROMID Study Group. J Clin Oncol. 2009;27:4656-63. doi:10.1200/JCO.2009.22.8510.

5. Kwekkeboom DJ, Krenning EP, Lebtahi R, Komminoth P, KosKudla B, de Herder WW, et al. ENETS consensus guidelines for the standards of care in neuroendocrine tumors: peptide receptor radionuclide therapy with radiolabeled somatostatin analogs. Neuroendocrinology. 2009;90:220-6. doi:10.1159/000225951.

6. Kwekkeboom DJ, de Herder WW, Kam BL, van Eijck CH, van Essen M, Kooij PP, et al. Treatment with the radiolabeled somatostatin analog [177Lu-DOTA0,Tyr3]octreotate: toxicity, efficacy, and survival. J Clin Oncol. 2008;26:2124-30. doi:10. 1200/JCO.2007.15.2553.

7. Imhof A, Brunner P, Marincek N, Briel M, Schindler C, Rasch H, et al. Response, survival, and long-term toxicity after therapy with the radiolabeled somatostatin analogue [90Y-DOTA]-TOC in metastasized neuroendocrine cancers. J Clin Oncol. 2011;29:241623. doi:10.1200/JCO.2010.33.7873.

8. Pfeifer AK, Gregersen T, Gronbaek H, Hansen CP, Muller-Brand J, Herskind Bruun K, et al. Peptide receptor radionuclide therapy with Y-DOTATOC and (177)Lu-DOTATOC in advanced neuroendocrine tumors: results from a Danish cohort treated in Switzerland. Neuroendocrinology. 2011;93:189-96. doi:10.1159/000324096.

9. Sabet A, Ezziddin K, Pape UF, Ahmadzadehfar H, Mayer K, Poppel $\mathrm{T}$, et al. Long-term hematotoxicity after peptide receptor radionuclide therapy with 177Lu-octreotate. J Nucl Med. 2013. doi:10.2967/ jnumed.112.119347.

10. Bodei L, Cremonesi M, Ferrari M, Pacifici M, Grana CM, Bartolomei $\mathrm{M}$, et al. Long-term evaluation of renal toxicity after peptide receptor radionuclide therapy with 90Y-DOTATOC and 177Lu-DOTATATE: the role of associated risk factors. Eur J Nucl Med Mol Imaging. 2008;35:1847-56. doi:10.1007/s00259-0080778-1.

11. Sabet A, Haslerud T, Pape U-F, Sabet A, Ahmadzadehfar H, Grünwald F, et al. Outcome and toxicity of salvage therapy with

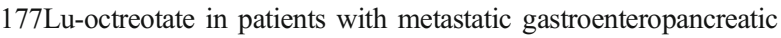
neuroendocrine tumours. Eur J Nucl Med Mol Imaging. 2013. doi: 10.1007/s00259-013-2547-z

12. van Essen M, Krenning EP, Kam BL, de Herder WW, Feelders RA, Kwekkeboom DJ. Salvage therapy with (177)Lu-octreotate in patients with bronchial and gastroenteropancreatic neuroendocrine tumors. J Nucl Med. 2010;51:383-90. doi:10.2967/jnumed.109. 068957.

13. Khan S, Krenning EP, van Essen M, Kam BL, Teunissen JJ, Kwekkeboom DJ. Quality of life in 265 patients with gastroenteropancreatic or bronchial neuroendocrine tumors treated with [177Lu-DOTA0,Tyr3]octreotate. J Nucl Med. 2011;52:1361-8. doi:10.2967/jnumed.111.087932.

14. Kahlert C, Kalluri R. Exosomes in tumor microenvironment influence cancer progression and metastasis. J Mol Med (Berl). 2013;91:431-7. doi:10.1007/s00109-013-1020-6. 